\title{
Two-Photon Continuous Flow Lithography
}

\author{
Simona C. Laza, Marco Polo, Antonio A. R. Neves, Roberto Cingolani, \\ Andrea Camposeo,* and Dario Pisignano*
}

The functional properties of polymeric particles strongly depend on their shape, size, and chemistry, making them useful in fields as diverse as photonics, ${ }^{[1]}$ microrheology, ${ }^{[2]}$ tissue engineering, ${ }^{[3]}$ and biomolecule analysis. ${ }^{[4]}$ For instance, polymer particles are used as carriers in drug delivery, ${ }^{[5]}$ where important issues related to particle flow, degradation and phagocytosis are influenced by their geometry and dimensions. ${ }^{[6]}$ Fundamental properties, including diffusion, suspension rheology, and selfassembly, can be effectively tailored by engineering the particle shape and composition. ${ }^{[7-9]}$ These findings motivate the development of novel fabrication techniques of micro- and nanoparticles with various shape and chemistry, ${ }^{[10,11]}$ possibly exhibiting low surface roughness and custom-designed three-dimensional (3D) geometry. To this aim, numerous methods have been proposed, including stretching of spherical particles, ${ }^{[12]}$ particle replication in non-wetting templates, ${ }^{[13]}$ photolithography, ${ }^{[14]}$ and microfluidic approaches. ${ }^{[2,15]}$ In particular, though originally limited to spherical or deformed spherical shapes, lab-ona-chip fabrication allows one to produce continuously particles with a wide range of morphologies and chemistries. ${ }^{[2]}$

The advent of continuos flow lithography $(\mathrm{CFL})^{[16]}$ from the Doyle's group has overcome some limitations of microfluidics, enabling the fabrication of complex structures..$^{[4,17-21]}$ In CFL, projection photolithography is generally used for the definition of the particle shape. Self-standing particles are obtained by photopolymerization of a pre-polymer sensitive to UV light during its flow through a microchannel $(\mu \mathrm{Ch})$, whereas chemical anisotropy is achieved by polimerizing across the interface of co-flowing pre-polymer solutions, since laminar flow provides poor, diffusion-limited mixing. Particles are synthesized in continuous runs, and their shape can be changed in real

Dr. S. C. Laza, Dr. M. Polo, Dr. A. A. R. Neves,

Dr. A. Camposeo, Prof. D. Pisignano

National Nanotechnology Laboratory of Istituto Nanoscienze-CNR

Università del Salento

via Arnesano, 1-73100 Lecce, Italy

E-mail: andrea.camposeo@nano.cnr.it; dario.pisignano@unisalento.it

Dr. A. Camposeo, Prof. D. Pisignano

Center for Biomolecular Nanotechnologies @ UNILE

Istituto Italiano di Tecnologia

via Barsanti, I-73010 Arnesano, Italy

Prof. D. Pisignano

Dipartimento di Ingegneria dell'Innovazione

Università del Salento

via Arnesano I-73100 Lecce, Italy

Prof. R. Cingolani

Italian Institute of Technology

via Morego 30, I-16163 Genova, Italy

DOI: 10.1002/adma.201103357 time using a digital mirror device (DMD) ${ }^{[22]}$ instead of standard masks. Multifunctional particles can also be produced by hydrodynamic focusing lithography. ${ }^{[21]}$ Overall, CFL methods developed to date achieve excellent results, however two important issues still remains open for this new class of techniques, namely the synthesis of (i) particles with sub-micrometer features and (ii) truly 3D particles in single-step processes. On one side, the highest reported resolution is $1.5 \mu \mathrm{m},{ }^{[10,22]}$ whereas the control of the particle structure at the sub-micrometer scale is often needed in many applications. On the other side, UV-mask projection and DMDs limit the achievable particle geometry to basically two-dimensional (2D) geometries.

The fabrication of 3D objects with arbitrarily complex shapes and feature sizes below the diffraction limit is instead possible by two-photon lithography (TPL). ${ }^{[23,24]}$ This process requires the simultaneous absorption of two photons having half the energy of the involved transition of the resist material, with probability depending on the squared intensity of the excitation laser. Two-photon absorption (TPA) is typically limited to a region very close to the laser focus, where light is intense enough to induce non-linear absorption, thus allowing to achieve subdiffraction spatial resolution. Photonic crystals, ${ }^{[25]}$ microtweezers and needles, ${ }^{[26]}$ and in situ scaffolding of cells ${ }^{[27]}$ are demonstrated by TPL. However, this technique on its turn is limited in throughput by its intrinsic seriality, making difficult to pattern large areas or to generate a high number of structures. Alternatives to circumvent such drawbacks include holography, ${ }^{[19,28]}$ though this method can not generate as arbitrary geometries as in TPL. In this work, we introduce a new approach for microfluidics-based production of polymeric particles, namely the two-photon continuous flow lithography (TP-CFL). This technique takes advantage of TPA high resolution to create objects with sub-micrometer and 3D features, and overcomes serial process limitations of traditional TPL by using multiple beam production under continuous flow. We demonstrate polymeric fibers, helical and bow-tie particles with subdiffraction resolution and surface roughness as low as $10 \mathrm{~nm}$.

A schematic view of the TP-CFL is shown in Figure 1a-b. In the following we indicate the direction of light propagation as $z$-axis, and the flow direction as $\gamma$-axis. In Figure $1 \mathrm{c}-\mathrm{f}$ we display some objects fabricated by TP-CFL. The 3D, free-standing helical structure shown in Figure 1d is obtained by the composition of the circular motion (diameter $20 \mu \mathrm{m}$ ) of the photopolymerization spot $\left(x_{0}, z_{0}\right)$, controlled by the piezo-stage perpendicularly to the flow direction ( $x-z$ plane), and the flow motion along the $\mu \mathrm{Ch}$ length ( $\gamma$-axis, Suppl. data movie 1). Particles with complex shape can be therefore synthesized by TP-CFL with a proper combination of flow conditions and writing-beam positioning within the $\mu \mathrm{Ch}$. This can be controlled either by moving the microfluidic device (as performed here) or by deflecting the 
(a)
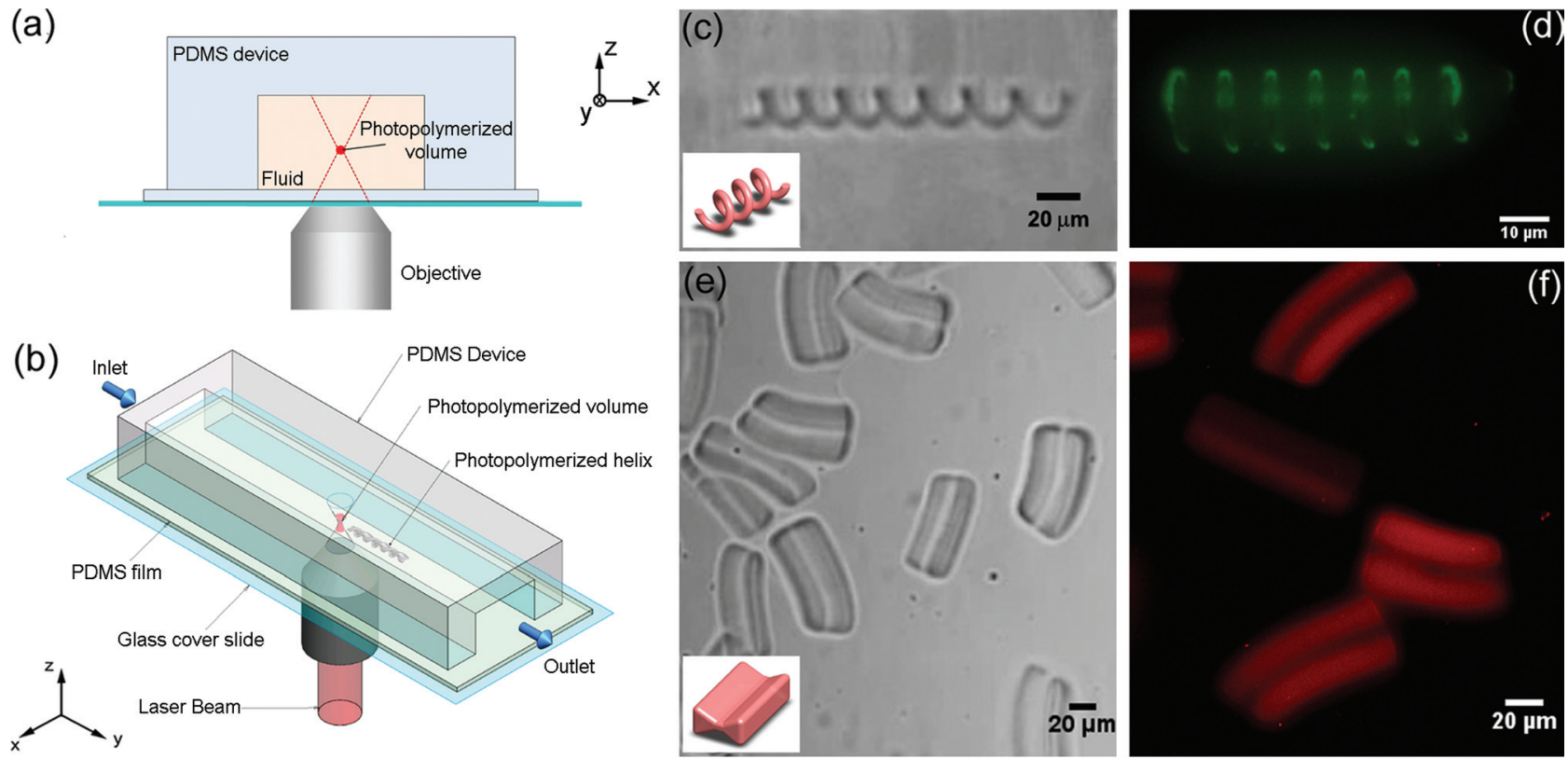

Figure 1. Cross-sectional (a) and top-view (b) scheme of the experimental setup. The coordinates values are $=0$ at the $\mu \mathrm{Ch}$ center $(x$ and $\gamma)$ and base $(z)$, respectively. (c-f) TP-CFL particles [schemes in the insets in (c) and (d)]. Bright field (c) and confocal fluorescence (d) micrographs of helices fabricated with a 1.3 NA objective (laser power $=150 \mathrm{~mW}$, flow rate $=0.2 \mu \mathrm{L} / \mathrm{min}$ ). Bright field (e) and confocal fluorescence (f) micrographs of elongated particles fabricated with a $0.45 \mathrm{NA}$ objective (laser power $=150 \mathrm{~mW}$, flow rate $=0.65 \mu \mathrm{L} / \mathrm{min}$ ).

writing beam within the channel using movable mirrors or DMD systems.

During writing, polymerization is initiated by radical formation following light excitation of the photoinitiator, which exhibits an absorption band at wavelength, $\lambda<430 \mathrm{~nm}$ (Supporting Information). The pre-polymer solution is instead transparent at the writing wavelength (785-800 nm), where absorption occurs by non-linear processes, as demonstrated by the intensity dependence measured by the Z-scan technique (Supporting Information). ${ }^{[29]}$ The low TPA cross section of the photoinitiator is well compensated by the high radical yield $(0.99),{ }^{[30]}$ making acylphosphine oxide molecules largely used in TPL. $[24,27,29,30]$

The particles shown in Figure 1e and 1f are produced by keeping the laser focal spot at a distance of $z_{0}=50 \mu \mathrm{m}$ from the glass substrate (Suppl. data movie 2). For a fixed flow rate, the particle length is determined by the shutter opening time (200-700 ms). These particles exhibit a distinct structure with two side lobes and a narrow central region, which is related to fundamental aspects of TP-CFL (Supporting Information). To investigate the correlation between the conditions of light focusing in the $\mu \mathrm{Ch}$ and the resulting particle shape, we determine the local distribution of the light intensity $(I)$ as resulting from using objectives with numerical aperture, NA $=0.45$ (Figure 2a) and 1.3 (Figure 2b). The calculation is performed neglecting polymer absorption and employing an analytical expression recently reported by us for the vectorial electromagnetic field of a monochromatic laser near the focus of a high NA objective, and through a refractive index mismatch. ${ }^{[31]}$ Figures $2 \mathrm{a}$ and $2 \mathrm{~b}$ display the logarithmic light intensity profile nearby the focal spot, in the plane $(x-z)$ containing the direction of propagation of the laser beam and perpendicular to the fluid flow inside the $\mu \mathrm{Ch}$, for a beam polarized along the $x$ axis. In Figures $2 \mathrm{c}$ and $2 \mathrm{~d}$, we display the intensity profile, calculated at the coordinate of the photopolimerization spot $\left(x=x_{0}\right)$, for objectives with $\mathrm{NA}=0.45$ and 1.3 , respectively, as a function of a spatial coordinate given by the shutter opening-time (displayed on top of panels $\mathrm{c}$ and $\mathrm{d}$ ), times the constant flow velocity, $v_{\text {flow. }}$. These plots provide a snapshot of the polymerized area in the plane $y-z$, and can be directly compared with the observed microobjects shapes. Indeed, the calculated intensity profiles correlate well to the corresponding particles shape (fluorescence micrographs in Figure 2e and Figure 2f). Highly focusing of the laser beam corresponds to an almost elliptical shaped focal spot (the so-called voxel, red area of Figure $2 \mathrm{~b}$ ), leading to almost cylindrical fiber-shape particles, as in Figure 2f. By contrary, lower NAs produce an area of almost uniform intensity more extended along the $z$-direction around the focal point, which origins the bow-tie shape of particles in Figures 1e-f and 2e. By a shutter opening-time of $10 \mathrm{~ms}$, we synthesize particles with diameter of about $400 \mathrm{~nm}$ (inset of Figure 2f), thus further demonstrating that TP-CFL can reach sub-diffraction resolution (Supporting Information). For sake of comparison, we recall that particles produced by confocal systems in $\mu$ Chs display typical sizes around $3 \mu \mathrm{m},{ }^{[32]}$ whereas sub-micrometer resolution, close to the diffraction limit, has been reported for techniques based on single-photon absorption by carefully exploiting the non-linear photoinitiator response. ${ }^{[33]}$

In conventional CFL, the polymerized volume roughly corresponds to the $\mu \mathrm{Ch}$ cross-section, and the synthesized particles do not stick to the channel walls because oxygen, diffusing through PDMS, inhibits the free-radical polymerization near the elastomer surface. ${ }^{[16,34]}$ Such anti-sticking effect is very important for the continuous transport of the polymerized objects by the 

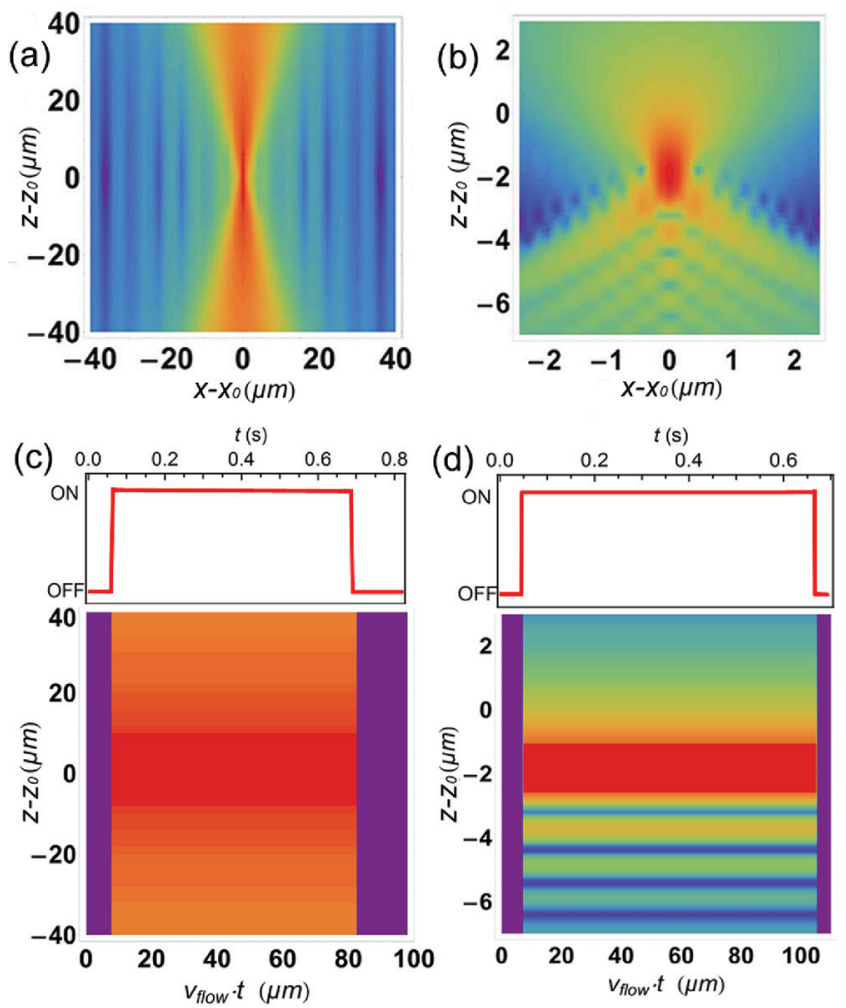

(e)
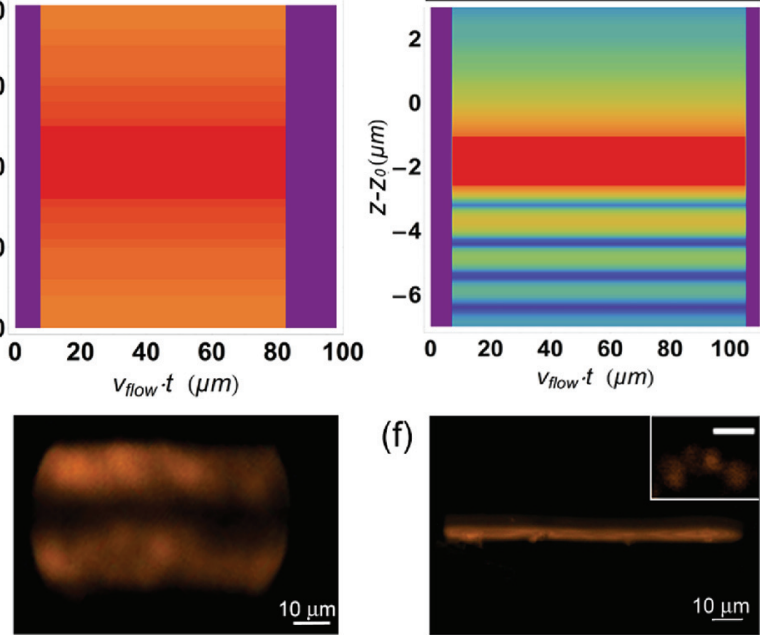

(f)

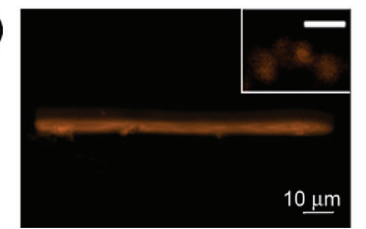

Figure 2. Calculated logarithmic intensity profile of the incident laser, along the axis of light propagation ( $z$ ), in the proximity of the focal spot for the $0.45 \mathrm{NA}$ (a) and 1.3 NA objective (b). Logarithmic intensity calculated at $x=x_{0}$ (transversal coordinate of the photopolymerization spot) vs shutter opening-time times flow velocity, for the $0.45 \mathrm{NA}$ (c) and 1.3 NA (d) objective. The shutter opening time is displayed on top of the panels (c) and (d), respectively. Confocal fluorescence images of resulting fibers by using the $0.45 \mathrm{NA}$ (e) and a $1.3 \mathrm{NA}$ objective (f). Scalebar: $10 \mu \mathrm{m}$. Incident laser power $=150 \mathrm{~mW}$. Flow rate $=0.25 \mu \mathrm{L} / \mathrm{min}$ and $0.40 \mu \mathrm{L} / \mathrm{min}$ for the $0.45 \mathrm{NA}$ and the $1.3 \mathrm{NA}$ objective, respectively. Inset: Confocal fluorescence image of sub-micrometric particles, fabricated by the 1.3 NA objective and shutter opening time $=10 \mathrm{~ms}$. Scalebar: $1 \mu \mathrm{m}$. Incident laser power $=200 \mathrm{~mW}$. Flow rate $=0.50 \mu \mathrm{L} / \mathrm{min}$.

flow, preventing $\mu \mathrm{Ch}$ clogging. Instead, in TP-CFL the polymerized volume is closely confined nearby the focal spot (few tens of $\mu \mathrm{m}$, depending on the objective NA) and the polymerized particles can be easily extracted from the synthesis region, thus opening the way to using materials with low gas permeability for chip fabrication. The possibility of polymerizing particles in different points within the $\mu \mathrm{Ch}$ volume is evidenced in Figure 3a-b. Multiple focused beams (generated by holographic systems or microlens arrays) can be used in parallel to enhance the throughput, by almost two orders of magnitude. A similar concept has been applied for the fabrication of anchored polymeric structures in conventional scanning TPL. ${ }^{[35]}$
Polymerizing particles in different $\mu \mathrm{Ch}$ points also allows to exploit the specific flow conditions present at different distances from the capillary walls. By a simplified description through a 2D Poiseuille microflow, the velocity profile is given by $v_{\text {flow }}(x)=\frac{\Delta P}{2 \eta L}\left(\frac{w}{2}-x\right)^{2}$, where $\Delta P$ indicates the pressure drop through the $\mu \mathrm{Ch}$ length. Since the length of the resulting fibers is directly related to $v_{\text {flow }}$, it can be controlled by moving the polymerization spot along the $\mu \mathrm{Ch}$ transversal coordinate $x$ in the $\mu \mathrm{Ch}$. Indeed, a Poiseuille law fits quite well the experimental fibers length, with some discrepancies for spot positions very close to the $\mu$ Chs lateral walls $(x= \pm w / 2)$, which are attributable to the $3 \mathrm{D}$ capillary geometry (deviations are expected $\cong$ $15 \%$ for our geometry), or to slipping conditions possibly occurring at the walls (Figure 3c). In particular, the fiber length depends almost linearly on the flow rate (Figure 3d). By tuning the flow rate and laser beam parameters, the particles dimensions can be dynamically changed during fabrication.

Finally, Figure 4 displays an Y-shaped $\mu \mathrm{Ch}$ with two flowing liquids, i.e. the pre-polymer (bottom inlet in Figure 4a) and a solution containing a fluorescent label (top inlet). As in other CFL methods, such laminar co-flow provides an almost sharp interface, with diffusion-limited liquid mixing. Therefore, Janus particles can be polymerized across the interface. ${ }^{[16]}$ Here we use TP-CFL by two beams to simultaneously produce two chemically-different helices (Figure 4b-f). Parallel processing of particles with different chemical compositions or anisotropy is indeed feasible in the same $\mu \mathrm{Ch}$.

In summary, TP-CFL is introduced as a flexible technique for the fabrication of sub-micrometer 3D polymeric particles. Fibers, bow-tie and helical particles are obtained with subdiffraction resolution. The fibers length can be controlled by the shutter opening-time and the fluid flow rate. Using multiple beams and laminar co-flows of different solutions allow the simultaneous fabrication of 3D particles with diverse chemical composition, which may find many applications in photonics and biotechnology.

\section{Experimental Section}

Pre-polymer solution: The pre-polymer solution consists of poly(ethylene) glycol diacrylate (PEG-DA, $M_{n}=700$, Sigma Aldrich) with $5 \%(\mathrm{v} / \mathrm{v}$ ) Lucirin TPO-L (ethyl-2,4,6-Trimethylbenzoylphenylphosphinate, BASF) as two-photon free radical photoinitiator. The solution shows an absorption band centered at $370 \mathrm{~nm}$ (full-width at half-maximum of $60 \mathrm{~nm}$ ) and a maximum absorption of $20 \mathrm{~cm}^{-1}$ (Supporting Information). Absorption at $\lambda=785-800 \mathrm{~nm}$ occurs mainly by TPA, as demonstrated by Z-scan (Supporting Information). This allows to synthesize particle with $3 \mathrm{D}$ shape since the absorption occurs in a volume close to the focal spot. The transparency of the pre-polymer solution at the laser wavelength also allows the polymerization in any point within the $\mu \mathrm{Ch}$ volume, and multiple-beam synthesis. Despite its low TPA cross section $\left(\sigma_{2} \sim 0.1-0.2 \mathrm{GM}\right.$ at $785-800 \mathrm{~nm}$, see Supporting Information and Ref. 29), Lucirin TPO-L is a highly efficient photoinitiator for twophoton applications, due to its high radical yield, ${ }^{[30]}$ reactive radical products and high solubility. ${ }^{[24,30]}$ The choice of the PEG-DA ${ }^{[16]}$ oligomer is motivated by its liquid form at room temperature (viscosity $\eta \cong$ $55 \mathrm{cP}$ ), well-assessed biocompatibility, and by the high polymerization rate of the acrylates. ${ }^{[27,36]}$ For fluorescence labeling the polymer, $0.005 \% \mathrm{wt}$ of methacryloxyethyl thiocarbamoyl rhodamine B (Polyscience Inc.) is added to the pre-polymer solution. 

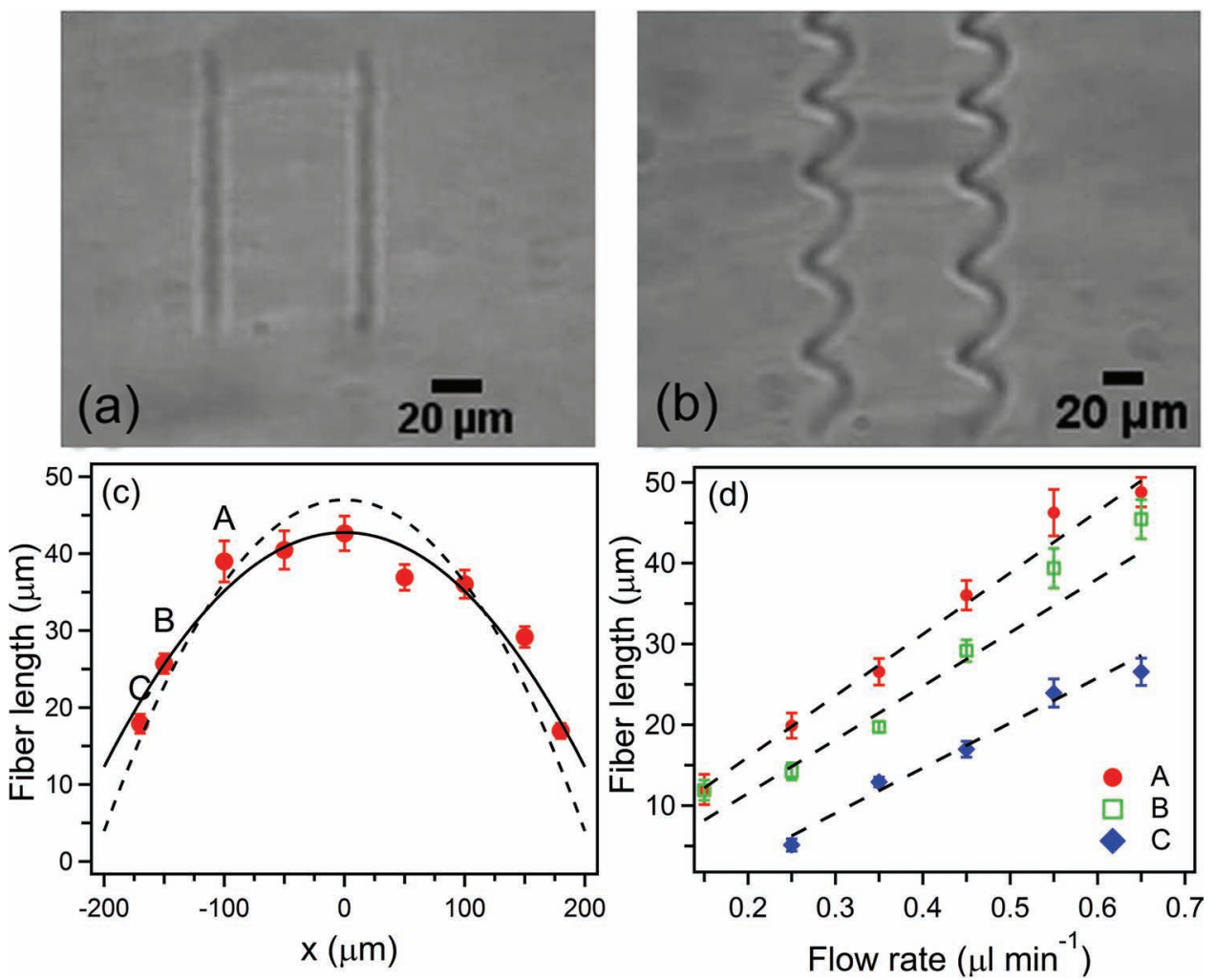

Figure 3. Bright field micrographs of (a) fibers and (b) helices produced by multiple beams. Laser power $=100$ (a) and $150 \mathrm{~mW}(\mathrm{~b})$, flow rate $=$ $0.20 \mu \mathrm{L} / \mathrm{min}$. The simultaneous polymerization in two flow points is achieved by splitting the original laser beam in two orthogonally polarized beams, then focused in the channel. (c) Fiber length vs. focal spot coordinate $(x)$ across the $\mu$ Ch. Data acquired at $z=50 \mu m$ from the channel base. Superimposed curve is the best fit by a parabolic Poiseuille profile of the microflow, by non-slipping conditions (dashed line) or non-zero velocity (continuous line) at the channel walls. (d) Fiber length vs. flow rate for different $x$ positions. A: $x=100 \mu \mathrm{m}, \mathrm{B}: x=150 \mu \mathrm{m}, \mathrm{C}: x=170 \mu \mathrm{m}$.
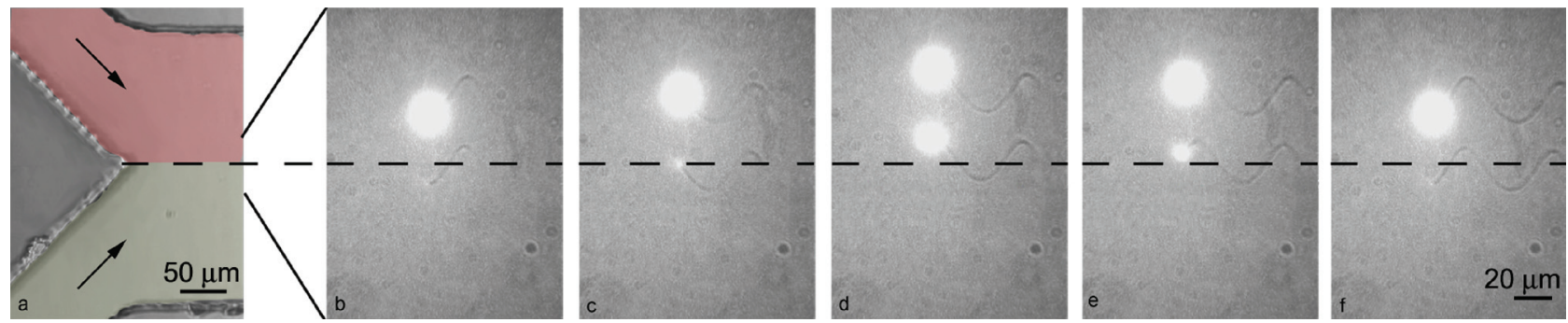

Figure 4. Parallel production of helices with single and multiple chemistries. (a) $\mu \mathrm{Ch}$ inlets. Scalebar $=50 \mu \mathrm{m}$. (b-f) Consecutive frames imaging helix fabrication process. Time between two frames $=150 \mathrm{~ms}(\mathrm{f})$. Scalebar $=20 \mu \mathrm{m}$. Laser power $=150 \mathrm{~mW}$, flow rate $=0.2 \mu \mathrm{L} / \mathrm{min}$. The focal spot (top one in b-f) is kept inside the fluorescence labeled stream producing a uniformly fluorescent helix, whereas a second spot (bottom one in b-f) periodically crosses the interface between the streams, thus generating helices with periodically distributed fluorescent segments. The top and the bottom spots exhibit continuous and intermittent luminescence, respectively, due to the Rhodamine emission under two-photon excitation (b- $f$ ).

Device Fabrication: The microfluidic devices, based on rectangular channels with length, $L=1 \mathrm{~cm}$, width, $w=400 \mu \mathrm{m}$ and height, $h=100 \mu \mathrm{m}$ are fabricated by replica molding from SU-8 master templates. The masters are realized by UV photolithography (15 s at $320 \mathrm{~W})$ on SU-8 2050 photoresist, spin-cast $(1700 \mathrm{rpm}, 30 \mathrm{~s})$ on $\mathrm{Si} / \mathrm{SiO}_{2}$. Elastomeric replicas of the master are obtained by pouring poly(dimethylsiloxane) (PDMS, Sylgard 184, Dow Corning) in 9:1 w/w base to curing-agent ratio, and by thermal curing at $75{ }^{\circ} \mathrm{C}$ for $15 \mathrm{~min}$. To obtain $\mu \mathrm{Chs}$, the elastomeric replicas are bonded with glass cover slides by oxygen plasma $(60 \mathrm{~s}$ at $70 \mathrm{~W})$. A thin layer of PDMS is spin-cast $(800 \mathrm{rpm}, 20 \mathrm{~s}$ ) on glass prior to bonding, and then cured at $75^{\circ} \mathrm{C}$ for $15 \mathrm{~min}$. For liquid injection and recovery, the two ends of the $\mu \mathrm{Ch}$ are punched to form inlet and outlet which are then connected via flexible tubes (i.d. $5 \mathrm{~mm}$ ) to a pumping syringe and to a collecting reservoir, respectively.

TP-CFL and particles characterization: The microfluidic device is clamped on a 3D multi-axes piezostage (P-562.3CD, Physik Instrument), mounted on an inverted microscope (Axiovert 40, Zeiss). A syringe pump (Harvard pump ' 11 ' Plus) supplies continuously the pre-polymer 
solution into the $\mu \mathrm{Ch}$ (typical flow rates in the range $0.15-0.65 \mu \mathrm{L} / \mathrm{min}$ ). The $\mathrm{TEM}_{00}$ laser beam from a Ti:Sapphire mode-locked laser oscillator (Coherent Mira 900, $\lambda=785-800 \mathrm{~nm}$, repetition rate $=76 \mathrm{MHz}$ and pulse duration $\cong 130 \mathrm{fs}$ ) is focused in the $\mu$ Ch through a $20 \times$ objective (Zeiss, Achroplan, NA $=0.45$ ), or through a $100 \times$ oil-immersion objective (Zeiss, Plan-Neofluar, NA = 1.3). A mechanical shutter controlled by a wave-generator is placed along the beam path to control the resulting length of the polymerized objects. The process is monitored in real-time by a charge-coupled device mounted on the microscope. All the reported laser powers are measured before the microscope entrance (almost $60 \%$ actually reaching liquid flows).

As typical of CFL methods, the flow in the microfluidic channel not only supplies the uncured material into the synthesis region, but also carries out the polymer particles. To collect free-standing, solid particles as those shown in Figures $1 \mathrm{~d}$ and $1 \mathrm{f}$, the mixture is diluted with ethanol in a 1:15 ratio, and then the polymerized micro-objects are obtained by sedimentation onto $1 \mathrm{~cm}^{2} \mathrm{Si} / \mathrm{SiO}_{2}$ substrates (oxide thickness $100 \mathrm{~nm}$ ). For sedimentation, many substrates are placed in parallel within a 24-well plate, filled with the diluted solution, for about two days. Before optical characterization, the samples are dried in vacuum.

Bright field images are collected by an up-right stereo-microscope equipped with a digital camera (Leica, DFC 490), and fluorescence micrographs of particles labeled with Rhodamine $B$ are obtained by a confocal microscope (Olympus, FV1000). The samples are excited by a diode laser emitting at $405 \mathrm{~nm}$, through an objective lens (40X) with NA= 0.85. A Multimode head equipped with a Nanoscope III a controller (Veeco) is used for tapping-mode atomic force microscopy (AFM), by Si cantilevers with resonance frequency of about $275 \mathrm{kHz}$.

\section{Supporting Information}

Supporting Information is available from the Wiley Online Library or from the author. The Supporting Information includes resist characterization, particles shape and roughness AFM analysis, movie clips of PEG-DA fibers and helices synthesis.

\section{Acknowledgements}

The authors are grateful to Dr. S. Girardo for device fabrication. The support of the Apulia Regional Strategic Project PS_144 is also acknowledged.

Received: August 31, 2011 Published online: February 3, 2012

[1] K. J. Stebe, E. Lewandowski, M. Gosh, Science 2009, 325, 159

[2] a) S. M. Anthony, L. Hong, M. Kim, S. Granick, Langmuir 2006, 22, 9812; b) S. Xu, Z. Nie, S. Xu, M. Seo, P. Lewis, E. Kumacheva, H. A. Stone, P. Garstecki, D. B. Weibel, I. Glitin, G. M. Whitesides, Angew. Chem. Int. Ed. 2005, 44, 724.

[3] Y. Du, E. Lo, S. Ali, A. Khademhosseini, Proc. Natl. Acad. Sci. USA 2008, 105, 9522.

[4] D. C. Pregibon, M. Toner, P. S. Doyle, Science 2007, 315, 1393.

[5] P. Horcajada, T. Chalati, C. Serre, B. Gillet, C. Sebrie, T. Baati, J. F. Eubank, D. Heurtaux, P. Clayette, C. Kreuz, J.-S. Chang, Y. K. Hwang, V. Marsaud, P.-N. Bories, L. Cynober, S. Gil, G. Férey, P. Couvreur, R. Gref, Nat. Mater. 2010, 9, 172.
[6] J. A. Champion, Y. K. Katare, S. Mitragotri, J. Control. Release 2007, 121, 3-9.

[7] Y. Han, A. M. Alsayed, M. Nobili, J. Zhang, T. C. Lubensky, A. G. Yodh, Science 2006, 314, 626.

[8] B. Madivala, S. Vandebril, J. Fransaer, J. Vermant, Soft Matter 2009, 5, 1717.

[9] S. C. Glotzer, M. J. Solomon, Nat. Mater. 2007, 6, 557.

[10] D. Dendukuri, P. S. Doyle, Adv. Mater. 2009, 21, 4071.

[11] S. Jiang, Q. Chen, M. Tripathy, E. Luijten, K. S. Schweizer, S. Granick, Adv. Mater. 2010, 22, 1060.

[12] J. A. Champion, Y. K. Katare, S. Mitragotri, Proc. Natl. Acad. Sci. USA 2007, 104, 11901.

[13] J. P. Rolland, B. W. Maynor, L. E. Euliss, A. E. Exner, G. M. Denison, J. DeSimone, J. Am. Chem. Soc. 2005, 127, 10096.

[14] A. B. D. Brown, C. G. Smith, A. R. Rennie, Phys. Rev. E 2000, 62, 951.

[15] W. Jeong, J. Kim, S. Kim, S. Lee, G. Mensing, D. J. Beebe, Lab Chip 2004, 4, 576

[16] D. Dendukuri, D. C. Pregibon, J. Collins, T. Allan Hatton, P. S. Doyle, Nat. Mater. 2006, 5, 365.

[17] J.-H. Jang, D. Dendukuri, T. Allan Hatton, E. L. Thomas, P. S. Doyle, Lab Chip 2007, 7, 818.

[18] K. W. Bong, D. C. Pregibon, P. S. Doyle, Lab Chip. 2009, 9, 863.

[19] J. H. Jang, D. Dendukuri, T. Allan Hatton, E. L. Thomas, P. S. Doyle, Angew. Chem. Int. Ed. 2007, 46, 9027.

[20] S. E. Chung, W. Park, S. Shin, S. A. Lee, S. Kwon, Nat. Mater. 2008, 7, 581.

[21] K. W. Bong, K. T. Bong, D. C. Pregibon, P. S. Doyle, Angew. Chem. Int. Ed. 2010, 49, 87

[22] S. E. Chung, W. Park, H. Park, K. Yu, N. Park, S. Kwon, Appl. Phys. Lett. 2007, 91, 041106.

[23] S. Kawata, H.-B. Sun, T. Tanaka, K. Takada, Nature 2001, 412, 697.

[24] C. N. LaFrata, J. T. Fourkas, T. Baldacchini, R. A. Farrer, Angew. Chem. Int. Ed. 2007, 46, 6238-6258.

[25] M. Deubel, G. Von Freymann, M. Wegener, S. Pereira, K. Busch, C. M. Soukoulis, Nat. Mater. 2004, 3, 444.

[26] S. Maruo, K. Ikuta, H. Korogi, Appl. Phys. Lett. 2003, 82, 133.

[27] P. Tayalia, C. R. Mendonca, T. Baldacchini, D. J. Mooney, E. Mazur, Adv. Mater. 2008, 20, 4494.

[28] S. Jeon, J.-U. Park, R. Cirelli, S. Yang, C. E. Heitzman, P. V. Braun, P. J. A. Kenis, J. A. Rogers, Proc. Natl. Acad. Sci. USA. 2004, 101, 12428.

[29] C. R. Mendonca, D. S. Correa, T. Baldacchini, P. Tayalia, E. Mazur, Appl. Phys. A. 2008, 90,633.

[30] T. Baldacchini, C. N. LaFratta, R. A. Farrer, M. C. Teich, B. E. A. Saleh, M. Naughton, J. T. Fourkas, J. Appl. Phys. 2004, 95, 11,6072 .

[31] A. A. R. Neves, A. Fontes, C. L. Cesar, A. Camposeo, R. Cingolani, D. Pisignano, Phys. Rev. E 2007, 76, 061917.

[32] Y. K. Cheung, B. M. Gillette, M. Zhong, S. Ramcharan, S. K. Sia, Lab Chip 2007, 7, 574.

[33] a) S. Maruo, K. Ikuta, Appl. Phys. Lett. 2000, 76, 2656; b) S. Maruo, K. Ikuta, Sens. Actuators A: Phys. 2002, 100, 70.

[34] D. Dendukuri, P. Panda, R. Haghgooie, J. M. Kim, T. A. Hatton, P. S. Doyle, Macromolecules 2008, 41, 8547.

[35] J. Kato, N. Takeyasu, Y. Adachi, H.-S. Sun, S. Kawata, Appl. Phys. Lett. 2005, 86, 044102.

[36] S.-H. Lee, J. J. Moon, J. L. West, Biomaterials. 2008, 29, 2962. 